\title{
醏
}

Jordi Espluga Bach*

Antonio García Rebollar**

\section{INNOVACIÓN EN COREA}

Corea puede ser un modelo a imitar en sus políticas de innovación, no solo por la cuantía de sus inversiones en investigación, desarrollo e innovación, sino por la originalidad de algunas de sus medidas de política económica y su rápida y eficaz implementación. En una comparativa internacional con datos de OCDE todavía presenta muchos defectos que corregir, pero la nueva política económica basada en el concepto de economía creativa está empezando a dar frutos en el sentido de avanzar en la convergencia de la industria con la cultura, la ciencia y la tecnología. En este contexto, aprendiendo de sus fortalezas y debilidades, la cooperación con España en materia de innovación resulta muy recomendable.

Palabras clave: economía creativa, cooperación tecnológica, internacionalización.

Clasificación JEL: O14, O32, O38, O53.

\section{Introducción}

Corea aparece hoy día como uno de los países líderes en innovación en el contexto mundial tras una experiencia de rápido desarrollo económico desde la década de los setenta. Su renta per cápita pasó de un escaso 12 por 100 de la renta de EEUU en 1970 al 62 por 100 en 2013.

Cuando se compara con España, existen muchas similitudes en cuanto al tamaño de su PIB y en población. De hecho, en declaraciones recientes del Fondo Monetario Internacional, en 2015 Corea ocupará el undécimo

\footnotetext{
* Delegado de CDTI. Embajada de España en Corea (Oficina Económica y Comercial).

** Consejero Económico y Comercial. Embajada de España en Corea (Oficina Económica y Comercial).
}

Versión de mayo de 2016. puesto en el ranking mundial de las veinte mayores potencias mundiales y España quedará en décimocuarto. En población, Corea llega a los 50 millones, mientras que España queda también por detrás con 47 millones de habitantes.

En los últimos diez años, el crecimiento en Corea ha sido más rápido que en España con tasas de crecimiento anuales que, hasta hace muy poco, superaban el 3 por 100 todos los años. Nuestro país perdió posiciones en la última década y una de las razones podría estar en un menor crecimiento de la productividad derivado de las inversiones en innovación.

En una comparación más rigurosa basada en los datos del Informe de Competitividad Global 2015-2016 del Foro Económico Mundial (World Economic Forum, WEF), Corea ocupa el puesto 26 y España el puesto 33 dentro de las economías más competitivas del mundo. 


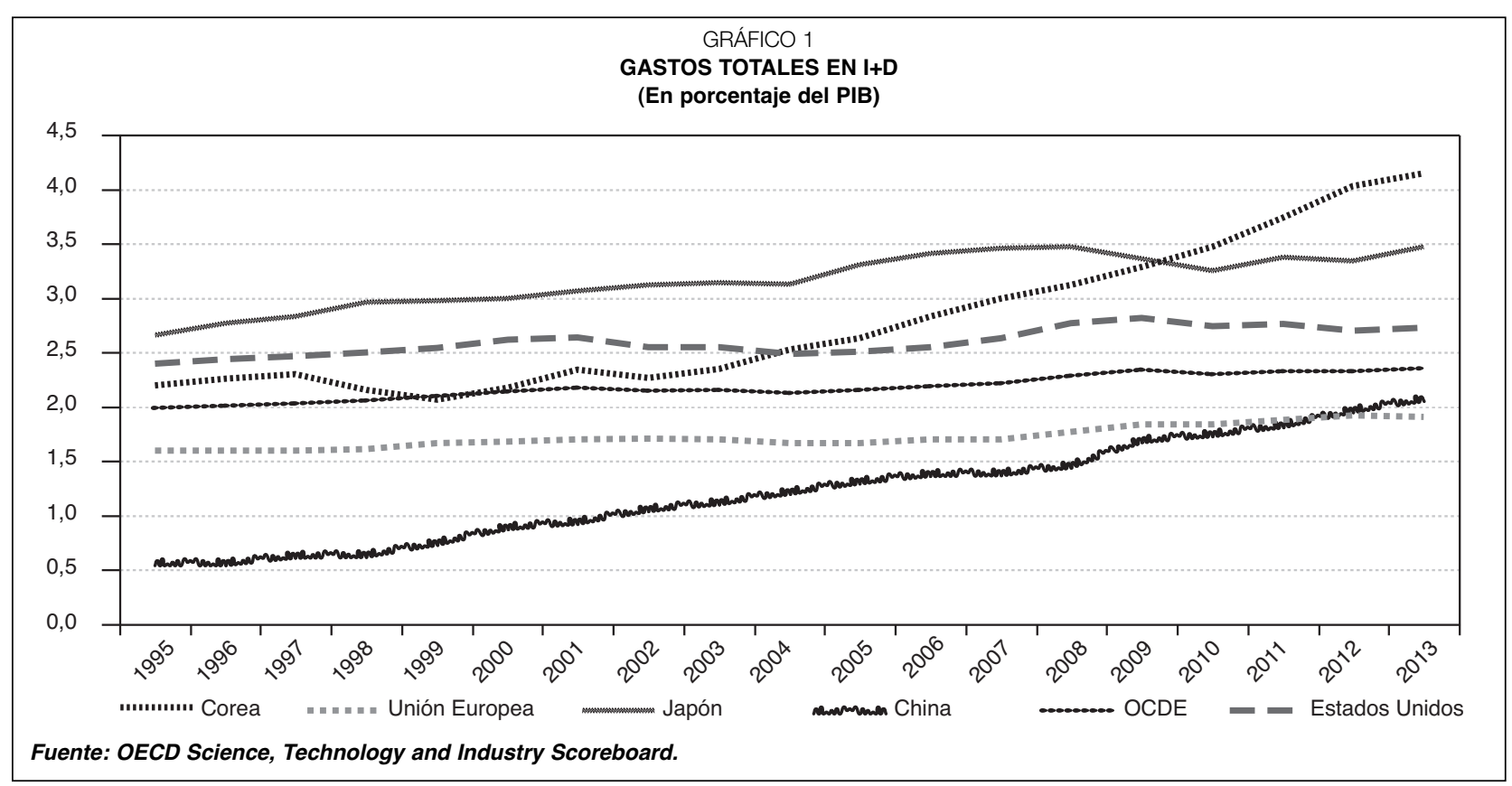

De todas formas, la comparativa en la que Corea destaca muy por encima de España es en su compromiso firme de apoyo a la investigación y desarrollo, de alrededor del 4,1 por 100 de su PIB, el mayor del mundo, y con un alto porcentaje de inversión privada.

Corea falla en aspectos como la estabilidad política debido al conflicto con Corea del Norte, en el excesivo peso de su burocracia y regulación o incluso en el acceso de las empresas a la financiación. Pero como ponían de manifiesto Álvarez Franco y Freijo García (2014), Corea supera con claridad a España en tres variables: la estabilidad macroeconómica, la educación y la innovación. En estas tres áreas, la puntuación de Corea es de las mejores del mundo.

Es difícil superar una economía sin déficit público y con una deuda pública por debajo del 40 por 100 del PIB. El superávit por cuenta corriente roza el 7 por 100 del PIB, es el sexto mayor exportador del mundo y todavía el tipo de interés básico del Bank of Korea se mantiene en el 1,5 por 100 . No hay desequilibrios macroeconómicos que puedan poner en dificultades a la economía coreana y Standard \& Poors le otorgó el año pasado la AA en su calificación crediticia, superando al rating de países vecinos como el propio Japón.
En educación, son líderes en los rankings de la OCDE por gasto privado, aunque la ventaja se debe sobre todo a los niveles básico e intermedio y no en la universidad. Los informes PISA colocan a los estudiantes coreanos en el primer nivel del mundo en matemáticas y en los cinco primeros puestos en ciencias y en literatura.

Este documento analiza el ecosistema de la ciencia y tecnología en Corea y sus iniciativas para potenciar la innovación en su modelo económico. El primer paso es estudiar cuáles son las fortalezas y debilidades de su sistema de innovación, seguido por un estudio de experiencias y medidas de política económica que pueden ser destacadas como buenas prácticas en el contexto internacional, y se acaba con un repaso de la colaboración con España.

\section{Fortalezas y debilidades del sistema de innovación}

En el Bloomberg Innovation Index de 2016, Corea es el número uno del mundo como el país más innovador por delante de Japón y $\triangleright$ 


\begin{tabular}{|c|c|c|c|c|c|c|c|c|c|}
\hline \multicolumn{10}{|c|}{$\begin{array}{c}\text { CUADRO } 1 \\
\text { INDICADORES DEL GASTO EN I+D }\end{array}$} \\
\hline Concepto & Año & Unidad & Corea & EEUU & Japón & Alemania & Francia & $\begin{array}{l}\text { Reino } \\
\text { Unido }\end{array}$ & China \\
\hline Gasto bruto total en I+D & 2013 & Miles de millones de USD & 60,5 & 457,0 & 170,9 & 106,4 & 62,6 & 43,5 & 191,2 \\
\hline Gasto bruto total en I+D per cápita...................... & 2013 & En USD & 1.079 & 1.444 & 1.342 & 1.296 & 950 & 679 & 141 \\
\hline Gasto bruto total en $\mathrm{I}+\mathrm{D}$ por investigador .......... & 2013 & Miles de USD & 175 & 361 & 259 & 296 & 236 & 168 & 129 \\
\hline 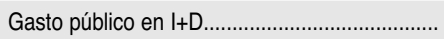 & 2013 & Porcentaje del gasto total & 24 & 35 & 24 & 30 & 37 & 33 & 21 \\
\hline Gasto de universidades en I+D......................... & 2014 & Porcentaje del gasto total & 9 & 14 & 14 & 18 & 21 & 26 & 7 \\
\hline Investigadores a tiempo completo ......................... & 2013 & Número de personas & 345.000 & 1.265 .000 & 660.000 & 360.000 & 265.000 & 259.000 & 1.484 .000 \\
\hline Investigadores a tiempo completo ......................... & 2013 & Por cada 1.000 ocupados & 13 & 8 & 10 & 8 & 9 & 8 & 2 \\
\hline 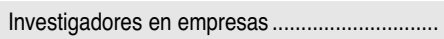 & 2013 & Porcentaje del total & 79,5 & - & 73,5 & 56,4 & 60,3 & 36,1 & 62,1 \\
\hline 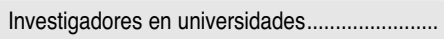 & 2013 & Porcentaje del total & 12,1 & - & 20,7 & 27,9 & 27,9 & 59,3 & 18,4 \\
\hline 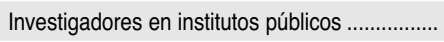 & 2013 & Porcentaje del total & 8,4 & - & 5,8 & 15,7 & 11,8 & 4,6 & 19,5 \\
\hline 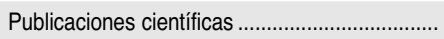 & 2014 & Número de trabajos & 54.691 & 391.850 & 77.552 & 103.765 & 71.720 & 110.578 & 253.633 \\
\hline Patentes PCT (Sistema internacional) .................. & 2014 & Número de solicitudes & 13.117 & 61.477 & 42.380 & 17.983 & 8.260 & 5.269 & 25.548 \\
\hline 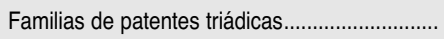 & 2013 & Número & 3.154 & 14.606 & 15.970 & 5.465 & 2.484 & 1.770 & 1.785 \\
\hline 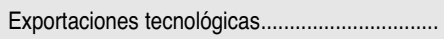 & 2013 & Miles de millones de USD & 9,8 & 126,5 & 34,8 & 66,6 & - & 38,8 & - \\
\hline 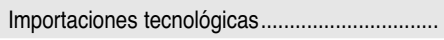 & 2013 & Miles de millones de USD & 15,5 & 87,6 & 5,9 & 54,2 & - & 12,7 & - \\
\hline Financiación pública del Gasto en I+D ............... & 2013 & Porcentaje del gasto total & 24,0 & 34,7 & 24,0 & 30,1 & 37,0 & 32,8 & 21,1 \\
\hline Financiación exterior del Gasto en I+D.............. & 2013 & Porcentaje del gasto total & 0,7 & 4,5 & 0,5 & 4,4 & 7,6 & 20,6 & 0,9 \\
\hline 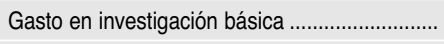 & 2013 & Porcentaje del gasto total & 17,6 & 16,5 & 12,6 & - & 24,2 & 15,5 & 4,7 \\
\hline Gasto en investigación aplicada ............................ & 2013 & Porcentaje del gasto total & 18,9 & 19,2 & 20,9 & - & 37,4 & 47,0 & 10,7 \\
\hline Gasto en desarrollo & 2013 & Porcentaje del gasto total & 63,5 & 64,3 & 66,5 & - & 38,4 & 37,5 & 84,6 \\
\hline Gasto privado en I+D con financiación pública. & 2013 & Porcentaje del gasto total & 5,6 & 9,2 & 1,3 & 4,6 & - & 8,1 & 4,5 \\
\hline 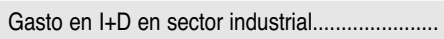 & 2013 & Porcentaje del gasto total & 88,9 & 65,8 & 88,7 & 86,1 & 50,2 & 40,0 & - \\
\hline 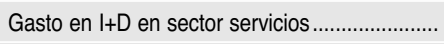 & 2013 & Porcentaje del gasto total & 8,3 & 28,6 & 10,0 & 13,1 & 46,9 & 58,1 & - \\
\hline 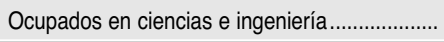 & 2012 & Porcentaje sobre total ocupados & 31,6 & 15,6 & 23,2 & 31,3 & 27,0 & 22,7 & - \\
\hline
\end{tabular}

Alemania. Para ello, se basan en datos de valor añadido industrial, número de empresas tecnológicas, nivel educativo, intensidad del gasto en investigación y desarrollo como porcentaje del PIB y número de patentes en relación a su población y PIB.

También es el número uno en el índice de desarrollo de las TIC o tecnologías de la información y comunicación que realiza la ITU (International Telecommunication Union) y fue publicado el pasado mes de noviembre con datos de 2014. Este análisis destaca a Corea como líder en el uso y conocimiento de las TIC $y$ en el acceso a Internet. No es de extrañar que, en el sector de las TIC, Corea sea el tercer mayor exportador del mundo, su peso represente el 33 por 100 de su exportación total y explique el 90 por 100 de su superávit comercial frente al resto del mundo.
En el análisis Innovation Union Scoreboard 2015 de la Comisión Europea de la innovación en la UE, destaca Corea como un país mucho más avanzado en materia de innovación. Lleva ampliando este margen respecto a los países comunitarios durante los últimos ocho años, ocupando la primera posición en su ranking por dos años consecutivos.

Corea también aparece como uno de los países más innovadores del mundo en el Global Inovation Index publicado en septiembre de 2015 en el informe elaborado por la Cornell University, INSEAD y la WIPO (World Intellectual Property Organisation) con 79 indicadores que resumen la actividad de cada país en la universidad, trabajos de investigación y número de patentes.

Cuando observamos el gasto total en investigación y desarrollo $(l+D)$ en relación al PIB de $\triangleright$ 
los últimos años, el resultado es impresionante: Corea es también el número uno por este concepto, con el 4,15 por 100 en 2013 según los datos de OCDE (2015). Solo Israel presenta una cifra comparable y se sitúa muy por delante de Japón con el 3,5 por 100.

Pero sorprende más aún el crecimiento de los últimos diez años en los que ha logrado adelantar a EEUU y a Japón en ese concepto. En 2014, KISTEP (2015) -Korean Institute of S\&T Evaluation and Planning- publica un dato de gasto total bruto en I+D de 63.700 miles de millones de wones coreanos (56.060 millones de USD al cambio actual) y un presupuesto del Estado en I+D para 2015 de 18.900 miles de millones de wones (16.630 millones de USD al cambio actual).

En el Cuadro 1 están resumidos los principales indicadores del gasto y resultados de la $I+D$ en un una comparativa internacional.

En general, el sistema de la I+D tiene poco que envidiar a los grandes países líderes en innovación, en relación a su tamaño, y se diferencia porque su mayor componente del gasto en I+D procede del gasto privado de las empresas y por tener una menor presencia de las universidades.

Además de ser fuertes en $1+D$, Corea cuenta con 11 industrias entre las 20 de mayor crecimiento global, especialmente en las tecnologías modernas de la información y las comunicaciones, semiconductores, óptica, materiales avanzados, tecnologías de la salud y en Internet de las cosas.

\begin{tabular}{|c|c|c|c|}
\hline \multicolumn{4}{|c|}{$\begin{array}{c}\text { CUADRO } 2 \\
\text { GASTO EN I+D POR SECTORES } \\
\text { (Millones de USD) }\end{array}$} \\
\hline & 2011 & 2014 & Porcentaje \\
\hline TIC & 16.830 & 21.939 & 34 \\
\hline Biotecnología..................... & 4.004 & 4.809 & 8 \\
\hline Nanotecnología ................ & 6.220 & 8.358 & 13 \\
\hline 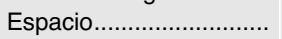 & 681 & 709 & 1 \\
\hline Medio ambiente ............... & 5.437 & 6.558 & 10 \\
\hline 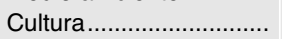 & 505 & 492 & 8 \\
\hline Otras..................... & 16.212 & 20.868 & 26 \\
\hline Total & 49.889 & 63.733 & 100 \\
\hline \multicolumn{4}{|c|}{ Fuente: Korea Main Science and Technology Indicators (KISTEP) } \\
\hline
\end{tabular}

Por sectores, el Cuadro 2 explica la composición del gasto donde no se aprecian grandes variaciones en los últimos años.

Casi el 80 por 100 de sus investigadores están en el sector privado empresarial, especialmente en las TIC que ocupan el 56 por 100 del gasto privado en I+D. Los ingenieros y científicos representan el 31,6 por 100 del total de ocupados, superando con claridad al resto de países avanzados.

El sector industrial juega un mayor papel que el sector servicios y está concentrado en las industrias más intensivas en $I+D$, que representaban el 60 por 100 del total de ocupados en 2013.

Se trata de una industria bien situada en las cadenas de valor mundiales con un alto grado de contenido de importaciones en sus exportaciones, el 41,6 por 100 en 2011. En algunas industrias como las TIC o automóviles llega hasta el 75 por 100.

Dentro del mundo de la propiedad intelectual, destaca por su elevado número de patentes en diseño industrial para las computadoras, equipos de comunicaciones y audiovisuales y otros equipos de informática.

A pesar del elevado peso del comercio tecnológico y de sus patentes en el exterior, una fuerte debilidad del sistema es la escasa internacionalización de su gasto en investigación y desarrollo, como ponen de manifiesto la baja financiación procedente del resto del mundo, solo el 0,7 por 100, cuando en países como el Reino Unido sobrepasa el 20 por 100, y sus bajos porcentajes de participación en proyectos internacionales de colaboración tecnológica, por debajo del 2 por 100 del total. Este dato se refleja, por ejemplo, en la baja participación de Corea en el Programa Marco (PM7) con 52 proyectos (países no comunitarios como Japón, Canadá y Australia tienen muchos más proyectos de colaboración).

Solo el 7 por 100 de las grandes empresas y el 4 por 100 de las pequeñas empresas innovadoras reconocen haber tenido algún tipo de $\triangle$ 
colaboración con el exterior. De todas formas, cabe decir que, en los últimos años, la Administración coreana está haciendo grandes esfuerzos para promover la participación de sus entidades en proyectos de colaboración en I+D internacional, como por ejemplo en el H2020 de la Unión Europea, con la que se ha llegado incluso a sincronizar convocatorias en diferentes tecnologías, así como la iniciativa Eureka para colaboración tecnológica industrial con entidades de Europa.

Corea acoge pocos estudiantes extranjeros en sus universidades y el único intercambio de científicos relevante procede de los ciudadanos coreanos que han estudiado o investigado en EEUU y que regresan a Corea. Este flujo positivo fue muy intenso en el periodo 2004 a 2008, pero es decreciente desde entonces. La Unión Europea ha tomado buena nota de esta baja movilidad de personal científico, y la ha puesto en sus prioridades de colaboración con Corea bajo el programa Marie Sklodowska Curie, precisamente para atraer a científicos coreanos para investigar en laboratorios europeos.

Tampoco se observa una intensa cooperación con otras empresas proveedoras o clientes, o con las universidades o centros de investigación dentro del propio país. Solo el 26 por 100 de las grandes empresas y el 15 por 100 de las pymes estuvieron involucradas en actividades conjuntas de innovación con el sector investigador.

En los procesos de internacionalización se observa además, en la actualidad, una dispersión y duplicidad de esfuerzos por parte de los ministerios, universidades e institutos de investigación públicos que actúan de forma descoordinada en la promoción comercial en el exterior y que no aprovechan suficientemente el potencial ni la red de oficinas disponible en su organismo principal para la internacionalización: la KOTRA o Korea Trade Investment Promotion Agency. Corea reconoce este problema, y desde hace un par de años ha abierto sus propios centros internacionales de apoyo a la comercialización de resultados de investigación y desarrollo (Korea Innovation Center), el primero fue en Bruselas y tiene por objetivos el apoyo al diálogo entre la UE y Corea, servir de plataforma de colaboración y networking en I+D, así como de apoyo a la transferencia tecnológica y en start-ups de base tecnológica. De forma similar el MOTIE (Ministerio de Comercio, Industria y Energía) ha abierto oficinas de promoción tecnológica industrial, en concreto en Bruselas, para la zona europea.

Corea cuenta con una economía con una alta concentración de poder de mercado en las grandes empresas y ello explica la mayor debilidad de su sistema de innovación e internacionalización. Estas grandes empresas colaboran poco entre ellas y menos aún con las pymes o con otras instituciones en el exterior.

El gasto privado en $I+D$ se concentra en un 52,1 por 100 en solo 10 empresas y en un 57,1 por 100 en 20 empresas. De todas ellas, destaca con claridad el grupo SAMSUNG que ha tenido un enorme crecimiento en los últimos años y que figura como la segunda mayor empresa del mundo en gasto en I+D, después de Volkswagen. De hecho, la cifra de 2014 publicada por la compañía llegó a los 13.800 millones de dólares, un 7,4 por 100 de sus ingresos totales y suponiendo prácticamente el triple de lo que gastó Apple en el mismo año o incluso próximo al total del gasto público del Gobierno de Corea en I+D.

Para explicar el gran peso de la I+D privada en Corea, una de las razones podría estar en la generosidad del sistema de deducciones fiscales en el Impuesto sobre Sociedades. En Deloitte (2015) se puede observar la comparación con otros impuestos de otros países. La definición de los conceptos de gasto y los porcentajes de deducción no difieren mucho de otros países, pero la inspección tributaria coreana se caracteriza por una interpretación más flexible y favorable a la empresa. 
Desde la perspectiva de las ayudas públicas, si bien existe una cierta disparidad de fuentes de financiación de proyectos nacionales, no ocurre lo mismo en la gestión y financiación de proyectos internacionales, centradas en dos agencias públicas, el NRF (National Research Foundation) y el KIAT (Korea Institute for Advancement of Technology) para colaboración científica y tecnológica respectivamente, y que ambas tienen en común las importantes ayudas en forma de subvención a disposición de los participantes coreanos en dichos proyectos internacionales.

En el área de Internet, Corea presenta uno de los mejores puestos del mundo en el acceso a través de ordenadores y sobre todo de los teléfonos móviles, con el 80 por 100 de sus habitantes usando Internet en 2014 y más del 80 por 100 a través del móvil, la tasa más alta en la OCDE. En 2014, Corea supera las medias de la OCDE del 95 por 100 de empresas con conexión a banda ancha y del 76 por 100 con página web. Sin embargo, el uso de la nube para almacenar datos y el comercio electrónico están por debajo del uso medio en los países de la OCDE. Solo el 10 por 100 de las pequeñas y el 24 por 100 de las empresas medianas en Corea usan la nube y el 15,7 por 100 de todas las empresas venden a través de Internet, frente al 21 por 100 en la OCDE. Por el contrario, las empresas coreanas destacan en el uso de aplicaciones complejas para la gestión de stocks, gestión de información (ERP) o la identificación por radiofrecuencia (RFID).

Además del escaso esfuerzo en innovación por parte de las pymes, otra de las debilidades del sistema radica en la poca presencia de la I+D en el sector servicios. Su peso en la economía es inferior al de otros países de la OCDE (59 por 100 del PIB), y en contraste con las grandes empresas industriales, su productividad es muy baja, aproximadamente la mitad.

Es cierto que, en el conjunto de la economía, el crecimiento de la productividad en los últimos siete años ha estado por encima del 3 por
100 superando a la media de países de la OCDE. Pero Corea todavía está al 55 por 100 de los niveles de productividad por hora trabajada que alcanzan los países más avanzados.

El crecimiento económico en Corea en el pasado ha estado basado fundamentalmente en una mayor dotación de los factores de producción y especialmente en el número de horas trabajadas. Por otra parte, no cabe duda de que en la última década la mayor inversión en I+D y en innovación está generando avances importantes en la productividad. Pero en el futuro no se podrá confiar en una mayor dotación de factores de producción, y el rápido envejecimiento de la población que se prevé para los próximos veinte años va a exigir que los crecimientos en la productividad sean aún mucho más intensos.

En las recomendaciones de la OCDE (2015) se hace especial énfasis en lograr un mayor apalancamiento del enorme esfuerzo que se realiza en el gasto en innovación. Para ello es necesario mejorar la productividad en el sector servicios, incorporar a las pymes en los avances del sistema de innovación, aumentar las transferencias de tecnología hacia las pymes y el sector servicios, aumentar los niveles de investigación en las universidades y aumentar la cooperación internacional en todos los ámbitos relacionados con la investigación, desarrollo e innovación.

\section{Buenas prácticas}

La presidenta Geun-hye Park ha impulsado desde 2013 el concepto de la economía creativa como bandera de todo su programa de reformas económicas. Aunque no existe una definición exacta de lo que se considera economía creativa, este concepto ha servido para poner la innovación en el centro de la agenda de las reformas económicas y a la vez para incluir otro tipo de medidas que refuercen la competitividad del país. 
Se trata de un concepto antiguo inspirado en los libros de John Howkins y que ya fue utilizado por otros países como el Reino Unido en 1997 en su campaña de "Creative Britain», aunque en este caso se trataba de una campaña solamente centrada en el sector servicios incluyendo la cultura, las finanzas, el arte y el diseño.

El objetivo principal de la economía creativa es muy claro: la creación de empleo. Aunque la tasa de desempleo global de la economía coreana no supera el 3,5 por 100 , existe en general la preocupación por las elevadas tasas que ha adquirido el desempleo juvenil. En realidad se trata de un problema de desajuste entre la oferta y la demanda de empleo, con unos jóvenes que gracias a su buena formación y cualificación buscan trabajar en los grandes conglomerados empresariales donde los niveles de sueldos son mucho más atractivos. Por el contrario, en el sector servicios y de las pymes existe una demanda de empleo insatisfecha. Por esta razón, en el marco de la economía creativa, se esperan potenciar nuevos sectores económicos, nuevas empresas y empleos mejor ajustados a la oferta de empleo juvenil. El énfasis se pone en la convergencia entre la industria y la ciencia, tecnología o incluso la cultura y se hace un llamamiento a la creatividad y a la imaginación en el desarrollo de nuevos negocios.

En el ámbito del presupuesto del Estado se ha creado una clasificación adicional para agrupar las dotaciones de los diferentes ministerios dirigidas a la economía creativa. Para 2015 la dotación total fue de 8.330 miles de millones de wones coreanos - unos 7.600 millones de USDcon un incremento del 17,1 por 100 respecto al presupuesto de 2014.

Dentro de la agenda de reformas económicas, puestas en marcha en los últimos tres años, existen numerosas medidas para favorecer la innovación y apalancar el gasto en investigación y desarrollo, a la vez que se pretende generar un entorno favorable para la creación de empresas y de nuevos emprendedores. Como principal impulsor de dichas medidas destaca el papel del Ministerio de Ciencia, Tecnologías de la Información y Planificación Futura (MSIP), ministerio que tiene asignadas las competencias en ciencia, y en el ámbito de las TIC en toda su cadena de valor, y que combinado con finanzas y tecnología se espera que sea un catalizador de proyectos tecnológicos en varias áreas industriales nuevas, como por ejemplo las fintech, un concepto que combina finanzas y tecnología y participado tanto por capital público como privado en los diferentes estadios de crecimiento de la empresa.

Hasta aquí la mayor parte de sus actuaciones económicas no se diferencian mucho de las medidas de reforma que se toman en numerosos países avanzados. Lo que resulta innovador en política económica es que bajo el concepto de la economía creativa incluyen otro tipo de medidas que afectan también a la política de educación, a la política industrial, social o cultural, medio ambiente e incluso a reformas en el mercado de trabajo o de política de competencia en los mercados de bienes y de reducción del poder dominante de los grandes grupos empresariales o política de defensa de la competencia. Al fin y al cabo, la política basada en la economía creativa se convierte en un cambio de paradigma que inspira todas las reformas estructurales que necesita la economía coreana para mejorar sus niveles de renta y bienestar en los próximos años.

Un ejemplo excelente de esta nueva política radica en la creación de 17 centros de economía creativa. En estos centros, el objetivo fundamental es la creación de nuevas empresas y el apoyo a emprendedores. Lo más novedoso es que en los centros no participan solamente el Gobierno central sino que incorporan a las grandes empresas, Gobiernos locales y asociaciones empresariales. Para cada centro se ha elegido un sector económico y una gran empresa $\triangleright$ 


\begin{tabular}{|c|c|c|}
\hline \multicolumn{3}{|c|}{$\begin{array}{c}\text { CUADRO } 3 \\
\text { CENTROS DE ECONOMÍA CREATIVA }\end{array}$} \\
\hline Región & Especialidad & $\begin{array}{l}\text { Empresa } \\
\text { responsable }\end{array}$ \\
\hline Seúl & Cultura y estilo de vida... & CJ \\
\hline Incheon & Logística y aviación .......................... & Hanjin \\
\hline Gyeonggi & Tecnologías de la información... & KT \\
\hline Chungnam & Energías limpias y agricultura .. & Hanhwa \\
\hline Daejeom & 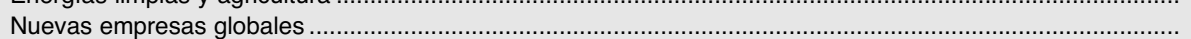 & SK Telecom \\
\hline Sejong & 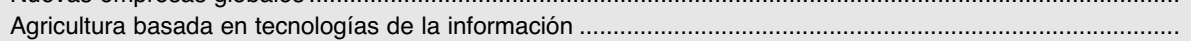 & SK Telecom \\
\hline Chungbuk & 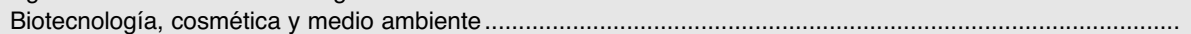 & LG \\
\hline Jeonbuk & Industria del carbón y cultura tradicional & Hyosung \\
\hline Jeonnam & 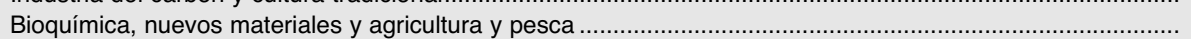 & \\
\hline Gwangju & 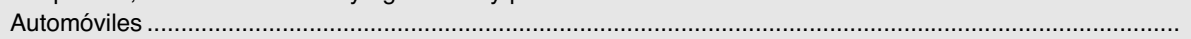 & Hyundai Motors \\
\hline Gyeongbuk & Nuevas fábricas inteligentes, cultura y agricultura & Samsung \\
\hline Daegu & Moda, maquinaria y partes de automóviles e industrias tradicionales............ & Samsung \\
\hline Ulsan & 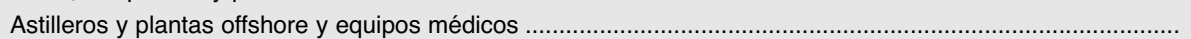 & Hyundai Heavy Industries \\
\hline Gyeongnam & Maquinaria innovadora & Doosan \\
\hline Busan & 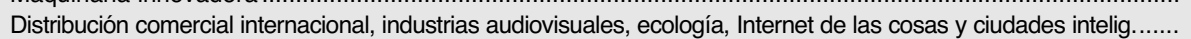 & Lotte \\
\hline Gangwon & Gestión y almacenamiento de datos, economía colaborativa, turismo, salud y granjas inteligentes ............ & Naver \\
\hline Jeju & 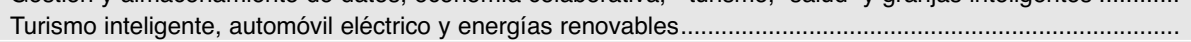 & Daum-Kakao \\
\hline
\end{tabular}

que actúa de socio principal en la financiación del centro. El centro proporciona equipos, componentes y asesoramiento legal, fiscal y comercial para que los emprendedores puedan acelerar el desarrollo de nuevas empresas o para que las pymes puedan poner en marcha nuevos proyectos empresariales.

Todos los centros han quedado inaugurados en el año 2015 y el número de empresas que se ha beneficiado de su apoyo se ha incrementado de 45 a 509 a lo largo de ese año. Para los próximos años se espera que más de 4.000 empresas puedan beneficiarse de estos programas.

El impulso simultáneo de los angel investors y de los fondos de capital riesgo ha permitido que el número de empresas financiadas a través de estos canales haya superado la cifra de 30.000 por primera vez en la historia. En 2013 el número de angel investors fue de 4.870 y la inversión en capital riesgo alcanzó el 0,1 por 100 del PIB, ocupando la tercera posición en los países de la OCDE tras Israel y Estados Unidos. El Estado juega un papel fundamental en la dotación de los fondos de capital riesgo con el 33 por 100 del total de activos en 2012 , a los que hay que sumar el 12 por 100 de los fondos públicos de pensiones.

Otras medidas de gran interés resultan del proceso de simplificación y concentración de las ayudas actuales a las pymes. En 2011 existían 201 programas de ayuda a pymes instrumentados por 14 ministerios del Gobierno central, 16 Gobiernos regionales y 130 organismos relacionados con las pymes. En la actualidad un solo ente público, la SMBC -Small Medium Business Corporation- se ha encargado de coordinar todas estas ayudas para evitar las duplicidades y mejorar su eficiencia. La SMBC cuenta con un presupuesto superior a los 8.000 de dólares para la ayuda financiera a través de créditos y subvenciones, la formación y consultoría y para la internacionalización de las pymes.

Los institutos públicos de investigación también van a dedicar una atención especial a poner sus trabajos al alcance de las pymes e incluso a ceder sus investigadores a las propias empresas para que la investigación se haga mucho más próxima a las necesidades reales del mercado. Por el momento, 233 investigadores han sido ya contratados por 225 empresas para llevar a cabo este tipo de actividades.

A las citadas actividades de desarrollo industrial e innovación se le suman las iniciativas científicas, donde destacan cinco áreas concretas clasificadas como prioritarias por el Gobierno: nanotecnología, tecnología espacial, fusión nuclear, células madre y energías limpias. 
Desde el año 2011, el Gobierno viene dedicando varios miles de millones de dólares a la nanotecnología y también recibe un gran apoyo por parte del sector privado. De hecho, casi la mitad de los trabajos de investigación publicados por Corea se realiza en el campo de la física y disponen de tecnologías punteras en áreas muy diversas como el grafeno para la electrónica flexible o en nanopartículas basadas en gelatina para asimilar las medicinas en el cerebro.

En tecnología espacial, el año 2013 supuso un hito importante con la puesta en órbita del primer satélite coreano $y$, en la actualidad, el Korea Aerospace Research Institute trabaja con éxito en el esfuerzo de crear un satélite tomando la órbita de la luna para su lanzamiento en el año 2020.

La investigación sobre fusión nuclear está en la línea de los países más avanzados en el mundo. Corea participa en el proyecto ITER europeo y cuenta con su propio reactor experimental de generación de plasma que comenzó sus operaciones en el año 2008. Los investigadores han comenzado el diseño de un nuevo reactor de fusión nuclear (K-DEMO) hace 3 años y esperan que pueda estar operativo en el año 2037 siendo los primeros en aspirar a conectar este tipo de reactores a la red eléctrica.

La investigación en células madre comenzó con el éxito de haber realizado la primera clonación de un perro y en la actualidad, bajo el liderazgo de la Universidad CHA, están consiguiendo grandes avances en el campo de la medicina regenerativa utilizando células madre a partir de células obtenidas de la piel de personas adultas.

Para el desarrollo de tecnologías que puedan utilizarse en la generación de energías renovables, el Gobierno ha comprometido 10.900 millones de dólares a los que se suman otros 8.000 millones de dólares que debe invertir el sector privado para los próximos ocho años. El gasto en investigación y desarrollo en esta área supera el 0,05 por 100 del PIB, por encima de la media de países de la OCDE. Además cuenta con deducciones fiscales en el Impuesto sobre Sociedades entre el 20 y el 30 por 100 de las inversiones realizadas en proyectos de investigación en energías renovables.

\section{Cooperación con España}

Dentro de las debilidades del sistema de innovación en Corea, la cooperación internacional sigue siendo una de las grandes asignaturas pendientes. Los esfuerzos del Gobierno son cada vez más intensos y están presentes en la agenda de todos los viajes internacionales de la presidenta Park y de sus ministros y altos cargos.

España cuenta con una oficina del CDTI (Centro para el Desarrollo Tecnológico Industrial) en Corea desde el año 1998 que ha tenido una colaboración continuada con su homólogo en Corea, el KIAT o Korea Institute for Advancement of Technology, una agencia pública bajo el MOTIE. España es uno de los pocos países que cuenta con un programa de cooperación tecnológica con carácter bilateral en base a los acuerdos ministeriales que amparan la colaboración entre CDTI y KIAT para la promoción de la colaboración tecnológica industrial entre entidades de España y Corea.

Actualmente la colaboración tecnológica entre España y Corea se canaliza bajo la iniciativa Eureka de la Unión Europea, una iniciativa que dada su flexibilidad, permite una definición ad hoc de convocatorias conjuntas conforme los intereses y estrategias de los países que la impulsan. Para el presente año se han publicado dos convocatorias bilaterales, con fechas de cierre en marzo y agosto, y como promoción se ha hecho uso del Innovation Week de Estocolmo donde se han celebrado encuentros empresariales entre España y Corea con delegaciones $\triangleright$ 
de ambos países y un total de 69 reuniones bilaterales. Fruto de estas iniciativas a lo largo de los pasados años, España ha pasado a ser el país, en el contexto europeo, con un mayor número de proyectos con Corea en el marco del programa Eureka, con un total de 37 proyectos certificados, y otros 23 adicionales bajo el programa KSI (programa previo al Eureka).

Esta cooperación se reforzó el pasado año con la renovación de los acuerdos ministeriales de colaboración con los ministerios coreanos del MOTIE (Industria) y MSIP (Ciencia) a raíz de la visita de la Secretaria de Estado de Investigación, Desarrollo e Innovación. Esta colaboración se verá este mes de mayo reforzada con la renovación del acuerdo entre CDTI y KIAT con ocasión de la visita del Presidente de KIAT a Madrid.

Además el Secretario de Estado de Comercio ha incluido el refuerzo en el presupuesto para la cooperación tecnológica con España en sus dos últimas agendas de política comercial en las comisiones mixtas ministeriales celebradas en los años 2014 y 2016. En esta última comisión se propuso incluir al instituto Korea Institute of Energy Technology Evaluation and Planning (KETEP), una agencia pública al cargo del desarrollo energético de Corea, y en especial, las energías renovables.

El ICEX comenzó en el año 2015 a participar con pabellones oficiales en ferias de los sectores de nanotecnología y de fusión nuclear facilitando el acceso a las empresas españolas al mercado de la ciencia y la tecnología en Corea. Por el momento han participado 15 empresas que han encontrado grandes posibilidades de negocio en el ámbito de la cooperación tecnológica, la transferencia de tecnología o la venta de equipos y componentes.

En el campo de las TIC o tecnologías de la información, el ICEX viene organizando anualmente un pabellón oficial en la feria de videojuegos G-Star de Busan desde el año 2013. Esta colaboración está dando sus frutos en el apoyo a emprendedores tecnológicos españoles que ven a Corea como un gran mercado para el lanzamiento de sus productos o para la cooperación en este campo.

Un área de gran interés en la cooperación actual con Corea es el área de atracción hacia España de inversiones en centros de I+D de sus grandes empresas. Samsung ya cuenta con un centro de estas características con más de veinte ingenieros en Madrid y Barcelona para el desarrollo de aplicaciones relacionadas con la tecnología móvil. Pero en otros países, como Polonia, tiene grandes centros con miles de ingenieros y nuestro país es un buen candidato para acoger este tipo de inversiones en la actualidad. EI ICEX-Invest in Spain participa todos los años en actividades de promoción con este objetivo.

En el ámbito privado, existen acuerdos de colaboración entre algunas asociaciones y universidades pero con una actividad muy reducida. En el Congreso de Aceleradores de Partículas IPC 2016 que se celebra en Busan este mes de mayo, la asociación española INEUSTAR firmó un acuerdo importante de este tipo con la KAFAT, asociación coreana para la energía de fusión nuclear y la tecnología de aceleradores de partículas. En este congreso participaron siete empresas españolas en un pabellón organizado por el ICEX que captó gran atención de los visitantes.

En el pasado, existía también un acuerdo de colaboración del 2007 entre el IDAE (Instituto español para la Diversificación y Ahorro de la Energía) y KEMCO (Korea Energy Management Corporation).

Podríamos citar muchos ejemplos de casos de éxito en el ámbito de la cooperación tecnológica entre empresas privadas. Por ejemplo, la Compañía Española de Sistemas Aeronáuticos (CESA) certificó un proyecto Eureka con la empresa coreana Em Korea Co. para la generación de gas inerte en las cabinas de aviones civiles, y cuya colaboración le ha llevado a estrechar lazos con su socio coreano Em Korea y a hacerse $D$ 
recientemente con un contrato de desarrollo de los componentes del sistema hidráulico del helicóptero LCH/LAH (Light Civil Helicopter/Light Armed Helicopter) de la KAI (Korean Aerospace Industries) con base en el EC155B1 de Airbus Helicopters.

Otros ejemplos de mención son: la instalación de un sistema de generación de oleaje multidireccional con fines experimentales donde la española VTI (Vázquez y Torres Ingeniería) cerró en 2015 una operación con la KRISO (Korean Institute of Ocean Science and Technology). En el sector espacial la participación de Thales España y la coreana Jungwoo Eng en el desarrollo de diversos componentes para los nuevos satélites que lanzará Corea en 2017 y 2018 y que estuvo financiado por CDTI y KIAT dentro del proyecto liderado por KARI (Korea Aerospace Research Institute).

\section{Conclusiones}

A pesar del enorme esfuerzo en inversiones en innovación de los últimos diez años, Corea todavía requiere mejoras en la productividad de su economía que le permita llegar a los niveles de renta y bienestar de las economías más avanzadas.

El Gobierno juega un papel crucial en este proceso y su plan de economía creativa está calando en la sociedad a la hora de poner la creatividad y a la innovación en los objetivos de los empresarios.

La fuerte concentración de poder en los grandes grupos empresariales ha permitido presentar grandes cifras de gastos en I+D y en innovación, pero falta una mayor cooperación con las pymes y con otras empresas del resto del mundo.

En este contexto, España cuenta con grandes oportunidades para reforzar su cooperación con Corea en ciencia y tecnología.

\section{Bibliografía}

[1] ÁlVAREZ FRANCO, V. y FREIJO GARCíA, J.M. (2014). «De la educación a la innovación: claves de la competitividad surcoreana». Boletín Económico de ICE, $\mathrm{n}^{\circ} 3051$, mayo 2014. Madrid: Ministerio de Economía y Competitividad.

[2] BLOOMBERG (2015). «The Bloomberg Innovation Index». Bloomberg Business. Disponible en

http://www.bloomberg.com/graphics/2015-ino vative-countries/ (Recuperado el 2 de mayo de 2016).

[3] CONNELL, S. (2013). «Building a creative economy in South Korea: analyzing the plans and possibilities for new economic growth». Academic Paper Series. Korea Economic Institute of America.

[4] DELOITTE (2015). 2015 Global Survey of $R \& D$ Tax Incentives.

[5] EUROPEAN COMMISSION (2016). Innovation Union Scoreboard 2015.

[6] JONES, R.S. (2014). «Fostering a creative economy to drive Korean growth». Economics Department Working Paper $\mathrm{n}^{\circ} 1152$, ECOMKP(2014)48. Paris: Oecd Publishing.

[7] KISTEP (2016). Korea main Science \& Technology Indicators, Center for International Affairs. Korea Institute of S\&T Evaluation and Planning.

[8] KONNECT (2015). Past and Future RTDI Analysis.

[9] MOON, M. (2015). «Understanding Compressed growth of Science and Technology in South Korea: Focusing on public Research Institutes". The Korean Journal for the History of Science, vol. 37, $\mathrm{n}^{\circ} 2$ (2015), pp. 431-453.

[10] MSIP (2015). Government R\&D Innovation Plan. Ministry of Science, Innovation and Planning.

[11] NATURE INDEX (2015). South Korea. Nature Index 2015 Asia-Pacific. Macmillan Publishers Limited.

[12] OECD (2015). «Policy Priorities For a Dynamic, Inclusive and Creative Economy». OECD Better Policies Series. Paris: Oecd Publishing.

[13] OECD (2015). "OECD Science, Technology and Industry Scoreboard 2015". Directorate for Science, Technology and Innovation. Paris: Oecd Publishing.

[14] WEF (2015). The Global Competitiveness Report 2015-2106. Geneva: World Economic Forum. 